MATHEMATICS OF COMPUTATION

Volume 76, Number 257, January 2007, Pages 385-388

S 0025-5718(06)01837-0

Article electronically published on September 14, 2006

\title{
DETECTING PERFECT POWERS BY FACTORING INTO COPRIMES
}

\author{
DANIEL J. BERNSTEIN, HENDRIK W. LENSTRA, JR., AND JONATHAN PILA
}

\begin{abstract}
This paper presents an algorithm that, given an integer $n>1$, finds the largest integer $k$ such that $n$ is a $k$ th power. A previous algorithm by the first author took time $b^{1+o(1)}$ where $b=\lg n$; more precisely, time $b \exp (O(\sqrt{\lg b \lg \lg b})) ;$ conjecturally, time $b(\lg b)^{O(1)}$. The new algorithm takes time $b(\lg b)^{O(1)}$. It relies on relatively complicated subroutines - specifically, on the first author's fast algorithm to factor integers into coprimes-but it allows a proof of the $b(\lg b)^{O(1)}$ bound without much background; the previous proof of $b^{1+o(1)}$ relied on transcendental number theory.

The computation of $k$ is the first step, and occasionally the bottleneck, in many number-theoretic algorithms: the Agrawal-Kayal-Saxena primality test, for example, and the number-field sieve for integer factorization.
\end{abstract}

Here is an algorithm that, given an integer $n>1$, finds the largest integer $k$ such that $n$ is a $k$ th power:

1. For each prime power $q$ such that $2^{q} \leq n$, write down a positive integer $r_{q}$ such that if $n$ is a $q$ th power then $n=r_{q}^{q}$.

2. Find a finite coprime set $P$ of integers larger than 1 such that each of $n, r_{2}, r_{3}, r_{4}, r_{5}, r_{7}, \ldots$ is a product of powers of elements of $P$. (In this paper, "coprime" means "pairwise coprime.")

3. Factor $n$ as $\prod_{p \in P} p^{n_{p}}$, and compute $k=\operatorname{gcd}\left\{n_{p}: p \in P\right\}$.

It is easy to see that the algorithm is correct. Say $n$ is an $\ell$ th power. Take any prime power $q$ dividing $\ell$. Then $n$ is a $q$ th power, so $n=r_{q}^{q}$; but $r_{q}$ is a product $\prod_{p \in P} p^{a_{p}}$ for some exponents $a_{p}$, so $n$ is a product $\prod_{p \in P} p^{q a_{p}}$. Factorizations over $P$ are unique, so $n_{p}=q a_{p}$ for each $p$. Thus $q$ divides $\operatorname{gcd}\left\{n_{p}: p \in P\right\}=k$. This is true for all $q$, so $\ell$ divides $k$. Conversely, $n$ is certainly a $k$ th power.

Take, for example, $n=49787136<2^{26}$. Compute approximations

$$
\begin{aligned}
& r_{2}=7056 \approx n^{1 / 2} \\
& r_{8}=9 \approx n^{1 / 8} \\
& r_{17}=3 \approx n^{1 / 17} \\
& r_{3}=368 \approx n^{1 / 3} \\
& r_{9}=7 \approx n^{1 / 9} \\
& r_{19}=3 \approx n^{1 / 19} \\
& r_{4}=84 \approx n^{1 / 4} \\
& r_{11}=5 \approx n^{1 / 11} \\
& r_{23}=2 \approx n^{1 / 23} \\
& r_{5}=35 \approx n^{1 / 5} \\
& r_{13}=4 \approx n^{1 / 13} \\
& r_{7}=13 \approx n^{1 / 7} \\
& r_{16}=3 \approx n^{1 / 16} \\
& r_{25}=2 \approx n^{1 / 25}
\end{aligned}
$$

Received by the editor July 2, 2004 and, in revised form, May 10, 2005.

2000 Mathematics Subject Classification. Primary 11Y16.

Initial work: Lenstra was supported by the National Science Foundation under grant DMS9224205. Subsequent work: Bernstein was supported by the National Science Foundation under grant DMS-0140542. The authors thank the University of California at Berkeley and the Fields Institute for Research in Mathematical Sciences. 
where $\approx$ means "within 0.6." Factor $\{49787136,7056,368,84,35,13,9,7,5,4,3,2\}$ into coprimes: each of these numbers is a product of powers of elements of $P=$ $\{2,3,5,7,13,23\}$. In particular, $n=2^{8} 3^{4} 5^{0} 7^{4} 13^{0} 23^{0}$, $\operatorname{so} k=\operatorname{gcd}\{8,4,0,4,0,0\}=4$. In other words, $n$ is a 4 th power, and is not an $\ell$ th power for $\ell>4$.

As discussed below, the literature already shows how to perform each step of this algorithm in time $b(\lg b)^{O(1)}$, where $b=\lg n$. Computing $n^{1 / k}$, which is used by some applications, also takes time $b(\lg b)^{O(1)}$.

Details of Step 1. Here is one of several standard ways to handle Step 1.

Given $n$ and $q$, use binary search and Newton's method to compute a floatingpoint number guaranteed to be within $2^{-32}$ of $n^{1 / q}$, as explained in [4, Sections 8 and 10]. The algorithms of [4 rely on FFT-based integer multiplication; see [6, Sections 2-4].

Define $r_{q}$ as an integer within $2^{-32}$ of this floating-point number. If no such integer exists, define $r_{q}=1$.

Each $r_{q}$ has $O(b / q)$ bits. Together the $r_{q}$ 's have $\sum_{q \leq \lg n} O(b / q)=O(b \lg \lg b)$ bits by Mertens's theorem. The algorithms of 4 take time $(\lg b)^{O(1)}$ per bit.

Another standard way to handle Step 1 is to define $r_{q}$ as an integer 2-adically close to $n^{1 / q}$, as explained in [4, Section 21].

One can change the bound $2^{-32}$. We caution the reader that the two numerical examples in this paper use different bounds. A smaller bound requires a higherprecision computation of $n^{1 / q}$ but-for typical distributions of $n$-is more likely to produce $r_{q}=1$, reducing the load on subsequent steps of the algorithm. The typical behavior of the algorithm is discussed below in more detail.

Details of Step 2. Given a finite set of positive integers, the algorithm of [5, Section 18] computes the "natural coprime base" for that set. The algorithm takes time $s(\lg s)^{O(1)}$ where $s$ is the number of input bits. The algorithm relies on FFTbased multiplication, division, and gcd; see 6, Sections 17 and 22].

Use this algorithm to compute the "natural coprime base" $P$ for $\left\{n, r_{2}, \ldots\right\}$. Together $n, r_{2}, \ldots$ have $O(b \lg \lg b)$ bits, so this takes time $b(\lg b)^{O(1)}$.

Details of Step 3. Given a finite coprime set $P$ of integers larger than 1 , and given a positive integer that has a factorization over $P$, the algorithm of 5 , Section $20]$ finds that factorization. The algorithm takes time $s(\lg s)^{O(1)}$ where $s$ is the number of input bits. The algorithm relies on FFT-based arithmetic.

Use this algorithm to factor $n$ over $P$. Together $n$ and $P$ have $O(b \lg \lg b)$ bits, so this takes time $b(\lg b)^{O(1)}$.

Competition. Previous work by the first author in [4] had already shown that $k$ could be computed in time $b^{1+o(1)}$. The algorithm of [4] computes $r_{q}$ for prime numbers $q$, and then computes several increasingly precise approximations to $r_{q}^{q}$, stopping when an approximation demonstrates that $r_{q}^{q} \neq n$.

The run-time bound for the algorithm in this paper has two advantages over the run-time bound for the algorithm in 4]:

- The new bound is smaller. The old bound was $b \exp (O(\sqrt{\lg b \lg \lg b}))$; the new bound is $b(\lg b)^{O(1)}$.

- The new proof requires considerably less background. The new proof relies on the first author's results in [5] on factoring into coprimes, but the old proof relied on deep results in transcendental number theory. 
The old algorithm is conjectured to take time $b(\lg b)^{O(1)}$, as discussed in 4, Section $15]$, but this conjecture seems very difficult to prove.

Performance in the typical case. For most values of $n$, computing a floatingpoint number within $2^{-32}$ of $n^{1 / 2}$ reveals immediately that $n$ is not a square, because the floating-point number is not within $2^{-32}$ of an integer.

Similarly, for almost all values of $n$, computing reasonably precise floatingpoint approximations to $n^{1 / 2}, n^{1 / 3}, \ldots$ reveals immediately that $k=1$. Here one can define "reasonably precise" as, e.g., "within $2^{-32} / b$." For example, take $n=3141592653589793238462643383$, and compute

$$
\begin{aligned}
& 56049912163979.2869928550892 \approx n^{1 / 2}, \\
& 1464591887.5615232630107 \approx n^{1 / 3}, \\
& 315812.9791837632319 \approx n^{1 / 5}, \\
& 8475.4793001649371 \approx n^{1 / 7}, \\
& 316.0391590557065 \approx n^{1 / 11}, \\
& 130.3663105302392 \approx n^{1 / 13}, \quad r_{13} \\
& 41.4456928612363 \approx n^{1 / 17} \\
& r_{17}=1 \\
& 28.0038933071808 \approx n^{1 / 19}, \quad r_{19}=1 \text {; } \\
& 15.6865795173630 \approx n^{1 / 23}, \quad r_{23}=1 ; \\
& 8.8751884186190 \approx n^{1 / 29}, \quad r_{29}=1 ; \\
& 7.7091205087505 \approx n^{1 / 31}, \quad r_{31}=1 \text {; } \\
& 5.5356192737976 \approx n^{1 / 37}, \quad r_{37}=1 ; \\
& 4.6844886605433 \approx n^{1 / 41}, \quad r_{41}=1 \text {; } \\
& 4.3598204254547 \approx n^{1 / 43}, \quad r_{43}=1 \text {; } \\
& 3.8463229122474 \approx n^{1 / 47}, \quad r_{47}=1 \text {; } \\
& 3.3022819333873 \approx n^{1 / 53}, \quad r_{53}=1 \text {; } \\
& 2.9245118649948 \approx n^{1 / 59}, \quad r_{59}=1 ; \\
& 2.8234034999139 \approx n^{1 / 61}, \quad r_{61}=1 \text {; } \\
& 2.5727952305908 \approx n^{1 / 67}, \quad r_{67}=1 \text {; } \\
& 2.4394043898716 \approx n^{1 / 71}, \quad r_{71}=1 \text {; } \\
& 2.3805279554537 \approx n^{1 / 73}, \quad r_{73}=1 ; \\
& 2.2287696658789 \approx n^{1 / 79}, \quad r_{79}=1 ; \\
& 2.1443267449321 \approx n^{1 / 83}, \quad r_{83}=1 \text {; } \\
& 2.0368391790628 \approx n^{1 / 89}, \quad r_{89}=1 \text {; }
\end{aligned}
$$

where now $\approx$ means "within $2^{-40}$." Evidently $k=1$.

For these typical values of $n$, there is no difference between the algorithm in this paper and the algorithm of [4. All the time is spent computing approximate roots. Doing better means computing fewer roots - see [4, Section 22] — or computing the roots more quickly; these improvements apply equally to both algorithms.

For the other values of $n$ - the atypical integers that are close to squares, cubes, etc. - the algorithms behave differently. It is not easy to analyze, or experiment with, the actual worst-case behavior of the algorithms, because it is not easy to find integers that are simultaneously close to many powers. We leave this as a challenge for the reader.

History. Bach, Driscoll, and Shallit in 2 introduced a quadratic-time algorithm to factor integers into coprimes. The obvious algorithm takes cubic time. 
Bach and Sorenson in [3] published various algorithms to detect perfect powers, i.e., to check whether $k>1$. One algorithm takes time $O\left(b^{3}\right)$. Another algorithm is conjectured to take time $O\left(b^{2} /(\lg b)^{2}\right)$ for most, but not all, $n$ 's.

The second and third authors of this paper observed in early 1994 that they could compute $k$ in time $O\left(b^{2}(\lg \lg b)^{2}\right)$ by factoring $n, r_{2}, \ldots$ into coprimes with the Bach-Driscoll-Shallit algorithm; recall that $n, r_{2}, \ldots$ together have $O(b \lg \lg b)$ bits. This line of work was abandoned several months later when the first author announced that $k$ could be computed in time $b^{1+o(1)}$ by the increasingly-preciseapproximations-to- $r_{q}^{q}$ method.

The first author later pointed out that this line of work deserved to be revived, since he had found an essentially-linear-time algorithm - see [5] - to factor integers into coprimes.

\section{REFERENCES}

[1] Eric Bach, James Dirscoll, Jeffrey Shallit, Factor refinement, in [7] (1990), 201-211; see also newer version [2]. URL: http://cr.yp.to/bib/entries.html\#1990/bach-cba MR.1231441 (94m:11148)

[2] Eric Bach, James Driscoll, Jeffrey Shallit, Factor refinement, Journal of Algorithms 15 (1993), 199-222; see also older version [1]. ISSN 0196-6774. URL: http://cr.yp.to/bib/entries.html\#1993/bach-cba MR.1231441(94m:11148)

[3] Eric Bach, Jonathan Sorenson, Sieve algorithms for perfect power testing, Algorithmica 9 (1993), 313-328. ISSN 0178-4617. MR1208565 (94d:11103)

[4] Daniel J. Bernstein, Detecting perfect powers in essentially linear time, Mathematics of Computation 67 (1998), 1253-1283. ISSN 0025-5718. URL: http://cr.yp.to/papers.html. MR.1464141 (98j:11121)

[5] Daniel J. Bernstein, Factoring into coprimes in essentially linear time, Journal of Algorithms 54 (2005), 1-30. ISSN 0196-6774. URL: http://cr.yp.to/papers.html\#dcba ID f32943f0bb67a9317d4021513f9eee5a. MR2108417

[6] Daniel J. Bernstein, Fast multiplication and its applications, to appear in Buhler-Stevenhagen Algorithmic number theory book. URL: http://cr.yp.to/papers.html\#multapps ID 8758803e61822d485d54251b27b1a20d.

[7] David S. Johnson (editor), Proceedings of the first annual ACM-SIAM symposium on discrete algorithms, January 22-24, 1990, San Francisco, California, Society for Industrial and Applied Mathematics, Philadelphia, 1990. ISBN 0-89871-251-3. MR1089882 (91i:68006)

Department of Mathematics, Statistics, and Computer Science (M/C 249), The University of Illinois at Chicago, Chicago, Illinois 60607-7045

E-mail address: djb@cr.yp.to

Mathematisch Instituut, Universiteit Leiden, Postbus 9512, 2300 RA Leiden, The NETHERLANDS

E-mail address: hwl@math.leidenuniv.nl

School of Mathematics, University of Bristol, Bristol, BS8 1TW, United Kingdom

E-mail address: j.pila@bristol.ac.uk 